essential for the gastrulation process. By culturing embryoid bodies containing wild-type and knockout Tet ESCs, the authors found that Tet-knockout cells had a higher level of DNA methylation in ectoderm enhancers, with no differences in the mesoderm and endoderm. Impaired demethylation was associated with an increase in early mesendoderm differentiation. However, Tet-knockout mesoderm cells showed impaired demethylation of mesoderm enhancers and were unable to differentiate into haematopoietic cells, which suggests that demethylation by TET is one of the drivers of lineagedefining enhancer demethylation. This finding implicates demethylation as an essential part of subsequent cell differentiation.

Overall, Argelaguet et al. show that pluripotent epiblast cells are 'primed' for differentiation into cells of the ectoderm lineage, which - the authors propose - may be a general feature of lineage commitment. Joseph Willson

ORIGINAL ARTICLE Argelaguet, R. et al. Multi-omics profiling of mouse gastrulation at single-cell resolution. Nature 576, 487-491 (2019)
Ten-eleven translocation (TET) methylcytosine dioxygenase enzymes demethylate enhancers and are molecular mechanisms) remains to be further investigated.

Given that ASOs were likely to be targeting RNAs while they were still being transcribed, both teams investigated whether the ASOs were impeding transcription. Both groups confirmed by chromatin immunoprecipitation (ChIP) of RNA polymerase II (Pol II) that the ASOs led to reduced Pol II occupancy downstream of the cleavage site, implying that the cleavage led to transcription termination.

A well-established model of transcriptional termination is the torpedo model. In this model, cleavage of a nascent transcript at the polyadenylation site creates a free $5^{\prime}$ end on the downstream transcript fragment, which provides an entry point for an exonuclease (particularly XRN2) to then 'catch up' and dislodge the transcribing Pol II. To assess whether such a mechanism occurs following ASO-mediated cleavage, both teams depleted XRN2 in their systems: Lee and Mendell used RNA interference, whereas Lai et al. used an engineered inducible degron. In both cases, XRN2 depletion almost completely prevented ASO-mediated transcription termination while still allowing RNA cleavage.

In the torpedo model, as XRN2 must catch up to Pol II to dislodge it, a cleavage event proximal to the polyadenylation site might not provide sufficient distance for XRN2 to terminate transcription prematurely. Indeed, both groups showed that ASOs targeted to the distal ends of genes resulted in transcript knockdown without concomitant transcription termination. This strategy could be valuable going forwards for separating the functions of an RNA from any roles of transcription of its locus.

Overall, these studies highlight dual roles of ASOs in transcript cleavage and transcription termination, which should be accounted for in the design and interpretation of functional studies.

Darren J. Burgess

ORIGINAL ARTICLES Lee, J.-S. \& Mendell, J. T. Antisense-mediated transcript knockdown triggers premature transcription termination. Mol. Cell https://doi.org/10.1016/j.molcel. 2019.12.011 (2020) | Lai, F. et al. Directed RNase $\mathrm{H}$ cleavage of nascent transcripts causes transcription termination. Mol. Cell https:// doi.org/10.1016/j.molcel.2019.12.029 (2020)
COMPARATIVE gENOMICS

\section{A primate view of gene expression}

The genetic changes that underlie human-specific traits remain largely obscure, although changes in gene regulation are known to play an important part. A comparative genomics study now catalogues gene expression and DNA methylation profiles of multiple tissues sampled from the same individuals across humans and two non-human primate species.

Catalogues that map similarities and differences in gene expression and its regulation between primate species have been useful to elucidate evolutionary adaptations. However, a confounding factor arises if multiple tissues are not sampled from the same individuals, as differences between tissues can be mistaken for differences between individuals. Blake, Roux et al. set to address this problem by using a balanced study design to compare gene expression and DNA methylation levels of heart, kidney, liver and lung tissue obtained from four humans, four chimpanzees and four rhesus macaques.

Gene expression levels were determined using RNA sequencing and estimated using an approach that was designed to minimize technical confounders and "biases driven by sequence divergence across species". Confirming previous findings, gene expression variation was greater across tissues than species, as determined by principal component analysis (PCA) and clustering analysis. The team then classified genes with tissue-specific expression patterns that were conserved across species and found that these genes had more interactions in transcription co-expression networks and more annotated protein-protein interactions than did genes with regulatory patterns that were not conserved or tissue specific, a finding that was even more notable when only looking at genes with expression patterns indicative of acting natural selection.

Analysis of DNA methylation, measured by low-coverage whole genome bisulfite sequencing (BS-seq), pinpointed 7,026-41,280 regions that were differentially methylated between tissues (within species) and were conserved across species. To analyse DNA methylation differences that might underlie gene expression differences across species and tissues, the team focused on orthologous genes for which expression and matching promoter DNA methylation data were available across species (7,725 genes in humans and chimpanzees; 4,155 genes for all three species). In brief, differences in promoter DNA methylation accounted for only a modest fraction of expression differences across species and tissues, with the greatest impact seen on genes with conserved tissue-specific expression patterns.

This comparative catalogue of gene regulatory phenotypes should be useful to decipher interspecies regulatory differences and how these map to biological and functional phenotypes in primates. In particular, looking at species-specific patterns of tissue-specific gene regulation may help to identify and characterize adaptations specific to humans.

Linda Koch

ORIGINAL ARTICLE Blake, L. E. et al. A comparison of gene expression and DNA methylation patterns across tissues and species. Genome Res. https://doi.org/10.1101/ gr.254904.119 (2020) 